versions of ice (the 'iceberg' and 'interstitial ice' models), as a 'self clathrate', or as a distorted four-fold coordinated network of hydrogen bonds.

The present consensus seems to be that liquid water is a macroscopic network of molecules connected by frequent but transient hydrogen bonds, which allow unbonded neighbours to occur in numbers that vary with temperature and pressure. Attempts to identify unambiguous patterns of local molecular order that represent portions of the known ice polymorphs have generally proved to be unproductive. In any case, several independent computer simulations for liquid water, using only molecular equations of motion and estimates of the fundamental intermolecular interactions, confirm the random, defective-network viewpoint while automatically producing the characteristic thermodynamic water anomalies.

Quantitative modelling variations on this network viewpoint underlie recent studies directed at an intriguing possibility: does the regime of supercooled liquid water harbour a hidden first-order phase transition between two metastable liquids with different densities (to which the term 'polyamorphism' has recently been attached), along with an associated second critical point?

Water that is internal to living organisms differs from its pure bulk form. Aqueous biological fluids are electrolytes, and typically contain a wide array of biopolymers, nutrients and metabolites. As Ball emphasizes, intracellular water is forced to occupy a very crowded neighbourhood indeed, with available channels between the biopolymers being measured in nanometres, if not in ångströms. Consequently, most biological water is surface, or interfacial, water. Yet this aqueous solvent medium has vital roles in controlling the native folding patterns of proteins, and acting as a lubricant for the entire dynamic apparatus of life.

Quantitative details of this solvation role are still a bit hazy, but some broad themes have emerged from research. In particular, Ball provides an account of the present understanding of the 'hydrophobic interaction' phenomenon, which contributes significantly to protein folding and to the structural stability of membranes by driving together hydrocarbon-like molecular moieties.

It is in connection with Ball's discussion of the interfacial properties of liquid water that I would raise a minor quibble with what otherwise appears to be an accurate text. This concerns the comparison of the range of hydrophobic surface effects to the diameter of human hair. By using random samples from my own head as a basis, I estimate that this statement appears to be out by about two orders of magnitude.

As an object of research scrutiny, water has arguably attracted more than its fair share of questionable, or even absurd, claims. But like it or not, these scraps of pathological science constitute a legitimate part of the biography of water, so Ball provides detailed accounts of three notorious examples: the 'polywater' episode, which began in the former Soviet Union in the 1960s; 'cold fusion', announced by Stanley Pons and Martin Fleischmann in the United States in March 1989; and the alleged homoeopathic phenomenon reported a year earlier by Jacques Benveniste and collaborators for repeatedly diluted solutions of anti-IgE antibodies. Perhaps the inclusion of the last of these was inevitable, given that Nature itself was an active but nervous participant in its dissemination.

Some might judge that these and other less prominent water aberrations detract from the scientific legitimacy of the field, but the author intimates correctly that the situation deserves a more positive spin. First, the responses to these challenges to technical common sense affirm the health and vigour of the scientific method. Second, occasional bizarre claims can be interpreted as far-out indicators that creative imagination is widely at play, and this, when suitably filtered, provides the driving force for progress.

I read this book while Hurricane Floyd was inflicting its epic watery damage on the Atlantic coastline of the United States. This was a forceful reminder that understanding, let alone predicting, phenomena of all scales in our water-rich environment is still woefully inadequate. The mind wanders into musing about what a sequel to this book written at the end of the next century might reveal that this volume cannot. But for now, Ball's contribution is a delightful status report.

Frank H. Stillinger is at Bell Laboratories, Lucent Technologies, 600 Mountain Avenue, Murray Hill, New Jersey 07974, USA.

\section{New rhythms of our lives}

\section{The 24 Hour Society}

by Leon Kreitzman

Profile: 1999.176 pp. 116.99

\section{Paolo Sassone-Corsi}

The moment that Neil Armstrong and Buzz Aldrin took humankind's first steps on the Moon, 30 years ago, characterizes one of the peaks of human technological progress. The event concerned everyone from an intellectual and moral point of view, but the technological impact on our everyday lives appeared minimal. In those days we had no fax, e-mail, Internet or mobile phones. Although we had the capacity to send men walking on the Moon, we were still very slow

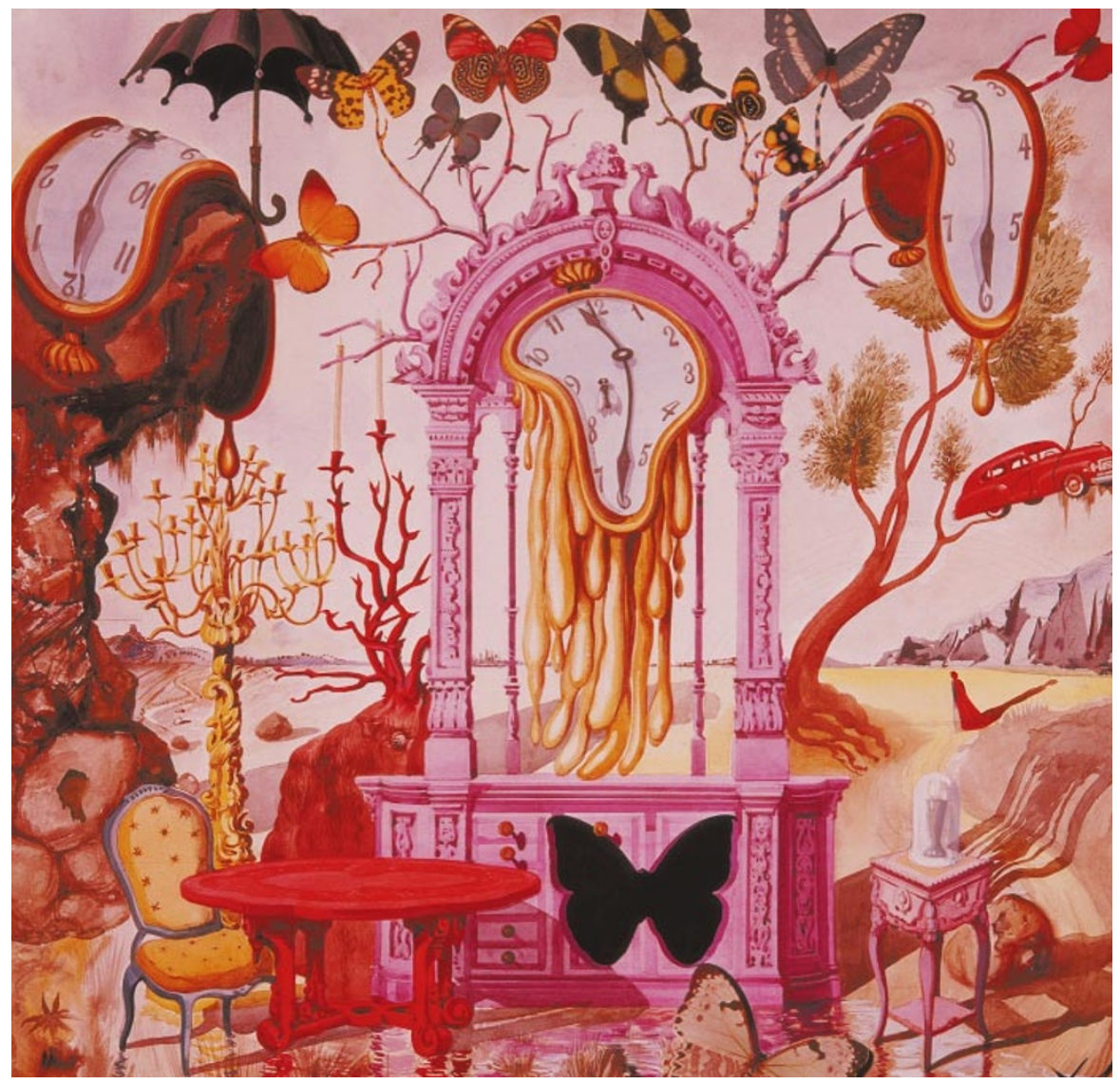

Dali on time: we take a more flexible view of the clock than did previous generations. 
and inaccurate in establishing long-distance contacts around our own planet. The revolution in communication and computing is a phenomenon of the past few decades. There have been so many dramatic changes in the way we communicate and travel worldwide that modern life has been profoundly affected.

The impetus behind the changes has been 'globalization'; a society has emerged which has an increasing need to communicate throughout the world, at any time of day or night; this is a society that can no longer operate on a $8 \mathrm{am}-5 \mathrm{pm}$ day. By enabling this society to be online 24 hours a day, says Leon Kreitzman, time has been expanded.

Scientists know this only too well. Trying to collaborate with a colleague based in California while in Europe, or arguing back and forth across the Atlantic with a scornful editor over a manuscript were difficult tasks only a few years ago. Time is even more pertinent to the business world, in which ignoring a 24-hour timescale would be financial suicide.

People adjust immediately to new devices, sometimes becoming conditioned to them without even knowing it. We have almost no recollection of how we functioned before faxes and e-mail, and contemporary society would clearly have a hard time functioning without these gadgets.

This is the core of Kreitzman's book, which is entertaining and easy to read, but also refined and comprehensive. Kreitzman ranges from business to informatics, from circadian biology to social science and working regulations. The 24 -hour society is most obviously represented by cities in Europe and the United States, but shopping and leisure are also available around the clock in Asia. The demand for flexible timing for these activities has developed in a growing proportion of the population. Of course, this is distinctive of a certain type of social economy, as Karl Marx (cited by Kreitzman) rightly reasoned: "labour during 24 hours of the day is the inherent tendency of the capitalist production."

Working mothers are a particularly apposite case, as most of them need to have a more stringent organization of their time because a high proportion of partners still do not help with housekeeping and child care. They are a group of people who can greatly benefit from a society that allows flexible use of facilities and services.

Another interesting example is the tourism industry, which has grown dramatically in the past couple of decades. Today tourism is global; in the 1980s it took over from oil as the world's largest industry, and now today it accounts for 10 per cent of the world's gross national product. People like to travel far afield, and as their time is limited, they want to have services, tourist attractions, museums, shopping and dining available at all times, everywhere they go.

Humans, distinct from all other animals, have had their circadian rhythms modified by social habits, electric light, television and international travel. Our natural sleep/wake cycle, as well as our endogenous biological clock, are continuously challenged by external stimuli that have nothing to do with the natural day/night astronomical cycle. Kreitzman gives a remarkably simple but clear (and correct) synthesis of our present knowledge of the biological clock. His hope is that "once we can control our rhythms many of the objections to shift-working and to the 24 Hour Society will fall away". The intrinsic danger of such thoughts is the possibility of biological control over people by society. But Kreitzman is careful to emphasize that advantages of the 24-hour society should not be "achieved by the exploitation of the health and safety of groups in the population".

So, is the 24-hour society some kind of present/future positive condition, or just a nightmare in which the Western world is caught up? Our parents say that it was so much better in their day, when the pace of life was slower and they had more time to enjoy valuable moments. Today it is difficult to have a normal conversation with a friend or colleague without being interrupted by a beep or a ring, and it does seem to many that enjoyable moments are becoming rarer. But I feel that Kreitzman is right; it is just a matter of getting adjusted to new rules and redefining temporal relationships. Then we will gradually, but continuously, learn to enjoy a different timing for the important things in our lives, and by the same token possibly gain time.

Paolo Sassone-Corsi is at the Institut de Génétique et de Biologie Moléculaire et Cellulaire, BP 163,

67404 Illkirch, Strasbourg, France.

\section{. Know thyself genetically}

\section{Genome: The Autobiography of a} Species in 23 Chapters

by Matt Ridley

Fourth Estate: 1999.752 pp. $£ 18.99, \$ 26$

\section{W. F. Bodmer}

Genetics, I believe, will stand alongside computing and nuclear physics as one of the outstanding areas of development by which this century will be remembered. The century started precisely with the rediscovery of Mendelism and it will finish with a first draft of the complete DNA sequence of the human genome. That remarkable catalogue of the human genes, ordered along the chromosomes to give us the Book of Man, will form the basis of most future biological and biochemical investigations of humans. The end of the Human Genome Project is the beginning of the real genetics of mankind. And this is the substantial challenge for understanding over the next century or more.

By combining the power of molecular genetics with classical population genetics and quantitative analysis, new approaches have been devised, not only for the 'positional' cloning of Mendelian traits whose biochemical basis was totally unknown, but also for identifying genes that contribute to the inherited susceptibility to multifactorial traits and diseases. Genes associated with nearly every Mendelian disease have been cloned, however rare the disease. But recall the great physician William Harvey, who urged us to treasure our exceptions, from which, as we now know, so much can be learnt. There is an exciting story to be told about genetics in the genomic era, but that is not to be found in Matt Ridley's book.

His title leads to the expectation that these exciting developments in genetics will be revealed to the general reader. However, in the preface we are told that "this is not a book about the human genome project", but instead "a book about what the project has found". The book is based on an intriguing idea, namely, to progress through the chromosomes chapter by chapter, using in each an example to illustrate what the genome reveals when it is properly read and interpreted. Ridley, a professional science writer and journalist, has, however, followed a common journalist's pathway and selected a collection of topics that he presumes will interest the reader whether or not they are relevant to his professed goal. Too many of these topics, such as sexual evolution, IQ, personality, stress and its relationship to immunity, ageing, memory and even, finally, free will, are simply not yet ready to yield to modern genetic approaches. They are mostly a basis for largely unsubstantiated speculation described in a way that could have been written without any reference to what the genome has to tell us.

Chromosome 1, the largest, is, according to Ridley, apparently empty, although a discussion of the rhesus blood groups might have been of interest. Chromosome 2 is used only as a vehicle for comparing Homo sapiens with the chimpanzee, the first of many "mind boggling" comparisons, in this case because of Ridley's apparent surprise that it may take only a relatively small number of differences in the DNA code to distinguish man from chimpanzees. Huntington's disease occupies chromosome 4. Certainly, it is an interesting story, but hardly, as the author claims, the most talked about genetic disease.

Given my own long-standing involvement with the HLA system and the genetics 